\title{
Bone mineral density, adiposity, and cognitive functions
}

\author{
Hamid R. Sohrabi 1,2,3, Kristyn A. Bates ${ }^{2,4}$, Michael Weinborn ${ }^{2,5}$, Romola S. Bucks ${ }^{5}$, \\ Stephanie R. Rainey-Smith ${ }^{1,2}$, Mark A. Rodrigues ${ }^{1,2}$, Sabine M. Bird ${ }^{2,3}$, Belinda M. Brown ${ }^{1,2}$, \\ John Beilby ${ }^{6,7}$, Matthew Howard ${ }^{2}$, Arthur Criddle ${ }^{8}$, Megan Wraith ${ }^{8}$, Kevin Taddei ${ }^{1,2}$, \\ Georgia Martins ${ }^{1,2}$, Athena Paton ${ }^{1,2}$, Tejal Shah ${ }^{1,2}$, Satvinder S. Dhaliwal ${ }^{9}$, Pankaj D. Mehta ${ }^{10}$, \\ Jonathan K. Foster ${ }^{11}$, lan J. Martins ${ }^{1,2}$, Nicola T. Lautenschlager ${ }^{3,12,13}$, Francis Mastaglia ${ }^{14}$, \\ Simon M. Laws ${ }^{1,2}$ and Ralph N. Martins ${ }^{1,2,3 *}$ \\ School of Medical Sciences, Edith Cowan University, Joondalup, WA, Australia \\ 2 The McCusker Alzheimer's Research Foundation, Nedlands, WA, Australia \\ ${ }^{3}$ School of Psychiatry and Clinical Neurosciences, University of Western Australia, Crawley, Australia \\ ${ }^{4}$ The School of Animal Biology, University of Western Australia, Crawley, WA, Australia \\ ${ }_{5}^{5}$ School of Psychology, University of Western Australia, Crawley, WA, Australia \\ ${ }^{6}$ School of Pathology and Laboratory Medicine, University of Western Australia, Nedlands, WA, Australia \\ 7 PathWest Laboratory Medicine of WA, Nedlands, WA, Australia \\ ${ }^{8}$ Western Medicine, Hollywood Specialist Centre, Nedlands, WA, Australia \\ ${ }^{9}$ School of Public Health, Curtin University of Technology, Perth, WA, Australia \\ ${ }^{10}$ Division of Immunology, Department of Developmental Neurobiolog, Institute for Basic Research in Developmental Disabilities, Staten Island, NY, USA \\ ${ }^{11}$ Neurosciences Unit, Health Department of WA, School of Psychology and Speech Pathology, Curtin University of Technology, Perth, WA, Australia \\ 12 Academic Unit for Psychiatry of Old Age, St. Vincent's Health, Department of Psychiatry, University of Melbourne, Parkville, VIC, Australia \\ ${ }_{13}$ The WA Centre for Health and Ageing, University of Western Australia, Crawley, Australia \\ ${ }^{14}$ Institute for Immunology and Infectious Diseases, Murdoch University, WA, Australia
}

\section{Edited by:}

Tania Álvarez Avellón, Universidad de Oviedo, Spain

Reviewed by:

Carsten Culmsee, University of

Marburg, Germany

Aurel Popa-wagner, Rostock Medical

School, Germany

\section{*Correspondence:}

Ralph N. Martins, School of Medical Sciences, Edith Cowan University,

270 Joondalup Dr, Joondalup, WA

6027, Australia

e-mail:ralph.r.martins@gmail.com
Cognitive decline and dementia due to Alzheimer's disease (AD) have been associated with genetic, lifestyle, and environmental factors. A number of potentially modifiable risk factors should be taken into account when preventive or ameliorative interventions targeting dementia and its preclinical stages are investigated. Bone mineral density (BMD) and body composition are two such potentially modifiable risk factors, and their association with cognitive decline was investigated in this study. 164 participants, aged 34-87 years old $(62.78 \pm 9.27)$, were recruited for this longitudinal study and underwent cognitive and clinical examinations at baseline and after 3 years. Blood samples were collected for apolipoprotein E (APOE) genotyping and dual energy $x$-ray absorptiometry (DXA) was conducted at the same day as cognitive assessment. Using hierarchical regression analysis, we found that BMD and lean body mass, as measured using DXA were significant predictors of episodic memory. Age, gender, APOE status, and premorbid IO were controlled for. Specifically, the List A learning from California Verbal Learning Test was significantly associated with BMD and lean mass both at baseline and at follow up assessment. Our findings indicate that there is a significant association between BMD and lean body mass and episodic verbal learning. While the involvement of modifiable lifestyle factors in human cognitive function has been examined in different studies, there is a need for further research to understand the potential underlying mechanisms.

Keywords: dual energy x-ray absorptiometry, cognition, apolipoprotein $E$, bone mineral density, episodic verbal memory, executive function, aging

\section{INTRODUCTION}

Dementia is a major debilitating disorder and a cause of significant concern for the currently aging population. In 2010, more than 35.6 million individuals were diagnosed with dementia worldwide (Prince et al., 2013). Prevalence projections indicate that dementia cases will dramatically increase worldwide by 2050 (Norton et al., 2014). In particular, a report from the Australian Institute of Health and Welfare estimates that the number of dementia patients in Australia will increase from 175,000 to 465,000 by the year 2031 (Australian Institute of Health and Welfare, 2007). Preventative research to reduce dementia-related burden is essential to tackle the financial as well as social consequences of this condition. Based on recent modeling reported by Alzheimer's Australia, if the onset of dementia was delayed by 2 years, a reduction of $13 \%$ or 398,000 cumulative new cases by 2050 would be achieved. Further, a delay of 5 years would reduce the number of cumulative new cases by $30 \%$, or 935,000 individuals by 2050 . Dementia-prevention programs would have a significant economic impact and improve the quality of life for affected individuals and their family (Vickland et al., 2012). Identification of potentially modifiable risk factors, including lifestyle factors, 
is a promising avenue for facilitating reductions in dementia incidence.

Dementia due to Alzheimer's disease (AD) is the most common form of dementia worldwide (Di Carlo et al., 2012). While the underlying causes of the late-onset form of the disease remain poorly understood, a complex mix of genetic, lifestyle, and hormonal factors is thought to contribute to the cerebral accumulation of a small peptide, beta amyloid (A $\beta$ ) (Butterfield et al., 2002; Isacson et al., 2002; Verdile et al., 2004; Wirths et al., 2004), resulting in the formation of extracellular amyloid deposits (Glenner and Wong, 1984; Masters et al., 1985). Research suggests that one third of AD cases are preventable (Norton et al., 2014). Because lifestyle and hormonal factors are potentially modifiable risk factors for $\mathrm{AD}$, they remain a focus of intense research scrutiny. One such hormone-related risk-factor is osteoporosis, which is defined as bone mineral density (BMD) more than 2.5 standard deviations below the mean for healthy adults aged between 20 and 40 years (W.H.O, 1994). The prevalence of osteoporosis increases with age. According to Osteoporosis Australia, 1 in 2 women and 1 in 3 men over the age of 60 will experience an osteoporotic fracture. In addition to age, female sex and menopause-related changes, previous fragility, previous fragility fractures, family history of hip fracture, and the use of oral corticosteroids are also significant risk factors for low BMD (Kanis, 2002; Finkelstein et al., 2008).

Osteoporosis and low BMD (osteopenia) have been associated with cognitive impairment and dementia (Lui et al., 2003; Rothman et al., 2007). BMD is regulated through the brain (Haberland et al., 2001; Karsenty and Oury, 2010), and this may partially explain the underlying relationship between BMD, cognitive dysfunction, and dementia. The brain regions involved in adiposity, (specifically the hypothalamus), also regulate bone remodeling through complicated and slow processes involving hormones including leptin (Haberland et al., 2001; Crockett et al., 2011). Leptin is thought to mediate BMD via binding to relevant receptors in the ventromedial hypothalamus (Haberland et al., 2001; Yang and Barouch, 2007) suggesting that osteoporosis may represent a neuro-skeletal condition (Takeda, 2009). Of note, plasma leptin level has been negatively associated with dementia and AD risk (Lieb et al., 2009). Additionally, the relationship between lower BMD and dementia may be modulated through cumulative exposure to estrogen as it was found in the Framingham Study, that lower femoral neck BMD was associated with a two-fold increase in risk of $\mathrm{AD}$ in women, potentially due to estrogen exposure (Tan et al., 2005).

Adiposity or body fat is another potentially modifiable risk factor associated with cognitive decline and dementia; however, research has produced somewhat conflicting results in this area. While most studies have supported a significant association between adiposity and cognitive decline (Luchsinger et al., 2007; Kerwin et al., 2011) other studies have failed to identify a significant relationship between these two on some of the cognitive functions associated with $\mathrm{AD}$, including verbal memory (Wolf et al., 2007). In some studies, adiposity has been associated with cognitive decline only in men (Kanaya et al., 2009), in individuals above 70 years old (Levine and Crimmins, 2012), or in participants below age 70 (Yoon et al., 2012). Adiposity is a risk factor for diabetes, hypertension, and cardiovascular changes; conditions which themselves contribute to significantly increased risk of AD (for a review see: Gustafson and Luchsinger, 2013) and cognitive decline due to vascular pathologies (Gustafson, 2012). For example, it has been suggested that adiposity, as a risk factor for insulin resistance and hyperinsulinemia may increase amyloid deposits in the brain resulting in $\mathrm{AD}$ (Luchsinger and Mayeux, 2007). In sum, the available evidence suggests that midlife central obesity plays a significant role in age-related cognitive decline and significantly increases the risk of dementia (Whitmer et al., 2008).

It is important to note that both increased adiposity and osteoporosis have been associated with cardiovascular disease (CVD) (Banks et al., 1994), which is associated with AD-plasma amyloid- $\beta$ protein (Bates et al., 2009) and has been shown to increase the risk of cognitive decline and dementia (Qiu et al., 2010; Norton et al., 2014). Interestingly, subclinical CVD increases the risk of bone loss and fracture (den Uyl et al., 2011) and BMD has been inversely associated with CVD (Farhat et al., 2007). Further, cardiovascular problems are associated with osteoporosis; moreover, lipid-related problems may play a role in increasing osteoporosis risk (Brown and Sharpless, 2004). Animal models support the association between osteoporosis and atherosclerosis (Parhami et al., 2000; Price et al., 2001). Observational studies have shown that higher atherogenic lipid profile and lipoproteins are inversely associated with bone density (Dimic et al., 2012; Sarkis et al., 2012) but the exact mechanisms underlying this relationship are unclear (Farhat and Cauley, 2008).

Cholesterol metabolism has been linked to apolipoprotein $\mathrm{E}$ epsilon 4 allele (APOE \&4), a major genetic risk factor for lateonset AD (Corder et al., 1993; Saunders et al., 1993; Roses, 1997). The ApoE protein is the major cholesterol transport protein in the brain, with allelic polymorphism in the APOE gene resulting in isoform-specific functional effects (e.g., higher risk of $\mathrm{AD}$ for $\varepsilon 4$ carriers and more resistance to $\mathrm{AD}$ in $\varepsilon 2$ carriers) (Weisgraber, 1994; Mahley et al., 1996). Some studies have indicated that, in addition to increased risk of $\mathrm{AD}$, APOE can also be involved in osteoporosis through mediating vitamin $\mathrm{K}$ transportation and/or inhibition of osteoblast differentiation (Kohlmeier et al., 1996; Parhami et al., 1997). However, a more recent study did not support the involvement of APOE genotype in BMD, increased bone loss, or higher risk of osteoporotic fractures (Schoofs et al., 2004).

The current study evaluated the relationship between adiposity, BMD and subsequent cognitive decline with respect to both screening of functional capacity and, more specifically, verbal episodic memory. We assessed BMD, adiposity and cognition, controlling for the potential effects of age, gender, and possession of the APOE $\varepsilon 4$ allele. Specific hypotheses included: (i) higher BMD would be significantly associated with better current and future cognitive functioning, particularly verbal memory; (ii) higher adiposity and lower lean body mass would be related to current cognitive function and predict subsequent cognitive function.

\section{MATERIALS AND METHODS STUDY DESIGN AND COHORT SELECTION}

One hundred and sixty four participants aged 34-87 years old $(62.78 \pm 9.27)$ were recruited from a larger, longitudinal, 
community-based study (the Western Australia Memory Study), investigating molecular and neuropsychological predictors of cognitive decline within younger and older adults (Clarnette et al., 2001; Sohrabi et al., 2009). The results were analyzed at baseline and after a 3-year follow-up. Participants completed annual blood and cognitive testing using standardized, validated screening and diagnostic measures. Exclusion criteria at the recruitment included: Mini Mental State Examination (MMSE) score $\leq 24$ (Folstein et al., 1975); clinically diagnosed dementia; untreated depression [Geriatric Depression Scale (GDS) score $\geq 11$ ]; history of neurological or psychiatric disorders affecting cognitive functions (e.g., stroke, Parkinson's disease, epilepsy, schizophrenia) and difficulty understanding or speaking English.

All participants provided written, informed consent to the study procedures including a body composition/BMD scan, using dual energy x-ray absorptiometry (DXA). Cognitive and clinical assessments, along with venous blood sampling occurred on the same day as DXA was performed. The study was approved by the Human Ethics Committees of Edith Cowan University, University of Western Australia, and Hollywood Private Hospital, Western Australia.

\section{DXA ANALYSIS}

The DXA technique quantifies bone mineral content by comparing the attenuation that occurs as a result of absorption of photons at two different energy levels, thereby creating a two dimensional BMD (aBMD) map (Van Loan and Mayclin, 1992). This analysis allows for the separation of body mass into bone, lean, and fat components.

In this study, DXA bone density and body composition scans were conducted on a central, whole body, Norland XR-46, pencil beam scanner using software version 4.1.1. The instrument was calibrated daily using a 77-step calibration standard QC phantom. The mean coefficient of variation (over 5 days) was 0.40 for BMD, 0.20 for lean mass and 0.14 for fat mass. Bone density measures were taken at the spine (L2-L4) and at the hip (femoral neck and total hip). The software generated: (i) $t$-scores, which compared each individual against a group, defined as possessing peak bone mass (i.e., normative data from healthy adults aged 20-40 years); and (ii) $z$-scores which compared an individual with data from their own age group.

Whole body scans were divided into regions of interest including head, chest, midriff, pelvis, and limbs. The measures taken included fat and lean mass (in $\mathrm{kg}$ ), total body fat percentage, Siri formula, and Brozek formula, for underwater weight equivalents (UWE) (Guerra et al., 2010). To estimate body fat \%, body mass density is calculated and converted to body fat \%, using the Siri or Brozek equations. These are the most commonly equations available (Guerra et al., 2010).

Most of the scores produced by DXA are highly intercorrelated. In order to address potential multicollinearity, we created composite, sample-based $z$ scores derived from the sum of all the $z$ scores calculated for lean mass and BMD raw scores, divided by the number of scores. The following raw scores were converted to $\mathrm{z}$ scores and divided by six (number of scores) to compute the composite lean mass $z$ score: Midriff lean mass + Pelvis lean mass + Left leg lean mass + Right leg lean mass +
Left arm lean mass + Right arm lean mass. The bone density composite $z$ score was calculated by summing the DXA Spine L2L4, Femoral Neck, and trochanter computed $z$ scores divided by three. We did not use a Fat mass composite $z$ score, but instead used the Siri UWE as this score is strongly associated with fat \% and other fat mass-related scores derived from DXA.

\section{CLINICAL AND COGNITIVE MEASURES}

Participants completed a comprehensive set of clinical and neuropsychological assessments lasting between 1.5 and $2.5 \mathrm{~h}$ and were offered breaks as needed. Depression at baseline was measured using the GDS (Yesavage et al., 1982). Premorbid cognitive ability was assessed using the Cambridge Contextual Reading Test (CCRT) (Beardsall, 1998). General cognitive functioning was assessed using the CAMCOG-R (Roth et al., 1998). Verbal episodic memory was assessed using the California Verbal Learning Test (CVLT) (Delis et al., 1988). Baseline and 3-year follow-up scores for the CVLT were calculated as follows: List Learning (List A; trials 1-5 total score), short delay free recall (SDFR), short delay cued recall (SDCR), long delay free recall (LDFR), long delay cued recall (LDCR), and recognition discriminability (RecDisc).

\section{BIOCHEMICAL AND GENETIC ANALYSIS}

On the same day as the DXA scan and cognitive/clinical assessment, a fasted venous blood sample was collected into serum, EDTA (containing prostaglandin E to prevent platelet activation) and heparin blood collection tubes (Interpath Services, Australia). The whole blood was then separated into different components using standard centrifugation techniques. DNA was isolated from leukocytes, and APOE genotype was determined via polymerase chain reaction (PCR) amplification and restriction enzyme digestion using the method originally described by Hixson and Vernier (1990), and outlined in Laws et al. (2002).

\section{STATISTICAL ANALYSIS}

Data were entered into Microsoft Excel and statistical analyses conducted using IBM SPSS Version 19 (IBM SPSS Statistics, 2010 New York, IBM Corp). After testing for normality, descriptive sample characteristics were analyzed. Next, a series of two-step hierarchical linear regressions were conducted in order to explore the relationships between biological variables, general cognitive function (as assessed by the CAMCOG-R) and episodic verbal memory (as measured by the above-mentioned CVLT sub scores). In step one, potential covariates including age at scan time, gender, APOE $\varepsilon 4$ allele status, and premorbid IQ (CCRT) were entered into the analysis. In step 2, variables derived from DXA were entered into the model. These variables included (1) Siri UWE Fat percentage, (2) Lean mass composite score, and (3) BMD composite score.

\section{RESULTS}

In the current study, participants included $69 \%$ women and $38 \%$ of participants were $A P O E \& 4$ carriers. Descriptive data for males/females, and APOE $\varepsilon 4+/ A P O E \varepsilon 4$ - groups are presented in Tables 1, 2. The percentage of participants who were $A P O E \varepsilon 4+$ did not differ by gender, $\chi^{2}(1 ; N=162)=0.010$, $p=0.919$. There were significant differences between men and 
Table 1 | Descriptive findings of dual energy x-ray absorptiometry (DXA) including the $p$-values corresponding to independent $t^{\dagger}$.

\begin{tabular}{|c|c|c|c|c|c|c|}
\hline & \multicolumn{3}{|c|}{ Sex } & \multicolumn{3}{|c|}{ APOE $\varepsilon 4^{e}$ Status } \\
\hline Age at scan & $63.65( \pm 7.88)$ & $62.39( \pm 9.85)$ & 0.423 & $63.31( \pm 9.36)$ & $61.97( \pm 9.21)$ & 0.372 \\
\hline Total fat $\%$ & $25.65( \pm 6.05)$ & $40.27( \pm 8.07)$ & $0.000 *$ & $36.63( \pm 10.36)$ & $34.30( \pm 9.61)$ & 0.155 \\
\hline Siri UWE fat \% & $21.14( \pm 5.67)$ & $32.71( \pm 7.35)$ & $0.000^{*}$ & $29.89( \pm 8.75)$ & $27.87( \pm 8.62)$ & 0.153 \\
\hline $\mathrm{HDL}^{\mathrm{c}}$ & $1.21( \pm 0.364)$ & $1.59( \pm 0.419)$ & $0.000 *$ & $1.46( \pm 0.431)$ & $1.50( \pm 0.458)$ & 0.570 \\
\hline $\operatorname{LDL}^{d}$ & $2.81( \pm 0.989)$ & $3.13( \pm 0.851)$ & $0.032^{*}$ & $3.01( \pm 0.878)$ & $3.08( \pm 0.959)$ & 0.626 \\
\hline Cholesterol & $4.93( \pm 0.992)$ & $5.44( \pm 0.885)$ & $0.001 *$ & $5.26( \pm 0.931)$ & $5.34( \pm 0.979)$ & 0.584 \\
\hline Triglycerides & $1.98( \pm 1.40)$ & $1.54( \pm 1.03)$ & $0.027^{*}$ & $1.69( \pm 1.17)$ & $1.64( \pm 1.19)$ & 0.798 \\
\hline
\end{tabular}

${ }^{\dagger}$ Equal variances not assumed; * $p<0.05$;

a Lean mass composite score included the DXA Lean mass Z scores for Midriff, Pelvis, Left leg, Right leg, Left arm, and Right arm divided by six;

${ }^{b}$ Bone Mineral Density Composite Score included the Z scores for DXA Spine L2-L4, Femoral Neck, and trochanter divided by three;

c High-density lipoprotein;

d Low-density lipoprotein;

${ }^{e}$ Apolipoprotein $E \& 4$.

Table 2 | Descriptive cognitive data including $p$-values corresponding to independent $\boldsymbol{t}^{\dagger}$.

\begin{tabular}{|c|c|c|c|c|c|c|}
\hline & \multicolumn{3}{|c|}{ Sex } & \multicolumn{3}{|c|}{ APOE $\varepsilon 4^{i}$ Status } \\
\hline CAMCOG $^{\mathrm{a}}$-baseline & $99.90( \pm 2.76)$ & $97.79( \pm 4.40)$ & $0.000^{*}$ & $98.72( \pm 3.50)$ & $97.98( \pm 4.91)$ & 0.270 \\
\hline CVLT List $A^{b}$-baseline & $53.71( \pm 10.37)$ & $54.61( \pm 11.20)$ & 0.625 & $55.05( \pm 10.83)$ & $52.98( \pm 11.07)$ & 0.244 \\
\hline CVLT SDFR' ${ }^{C}$-baseline & $10.75( \pm 2.86)$ & $11.20( \pm 2.91)$ & 0.350 & $10.99( \pm 2.98)$ & $11.10( \pm 2.73)$ & 0.817 \\
\hline CVLT LDFR ${ }^{\mathrm{e}}$-baseline & $11.22( \pm 2.77)$ & $11.17( \pm 3.04)$ & 0.924 & $11.32( \pm 2.92)$ & $10.87( \pm 2.96)$ & 0.340 \\
\hline CVLT LDCR ${ }^{f}$-baseline & $11.61( \pm 2.81)$ & $11.42( \pm 3.23)$ & 0.727 & $11.59( \pm 3.06)$ & $11.23( \pm 3.15)$ & 0.475 \\
\hline CVLT RecD9-baseline & $94.77( \pm 5.96)$ & $93.26( \pm 10.76)$ & 0.354 & $93.70( \pm 11.11)$ & $93.66( \pm 6.36)$ & 0.981 \\
\hline CAMCOG-F/Uh & $98.36( \pm 4.67)$ & $98.12( \pm 4.23)$ & 0.763 & $98.30( \pm 3.96)$ & $97.98( \pm 5.02)$ & 0.682 \\
\hline CVLT List A-F/U & $56.16( \pm 9.77)$ & $62.09( \pm 10.41)$ & $0.002^{*}$ & $60.41( \pm 10.39)$ & $59.98( \pm 11.01)$ & 0.818 \\
\hline CVLT SDFR-F/U & $11.30( \pm 2.89)$ & $12.43( \pm 2.78)$ & $0.028^{*}$ & $11.98( \pm 3.01)$ & $12.25( \pm 2.61)$ & 0.588 \\
\hline CVLT RecD-F/U & $95.02( \pm 4.83)$ & $96.04( \pm 6.95)$ & 0.380 & $95.83( \pm 4.86)$ & $95.50( \pm 8.40)$ & 0.766 \\
\hline
\end{tabular}

${ }^{\dagger}$ Equal variances not assumed; ${ }^{*} p<0.05$;

${ }^{a}$ The Cambridge Cognitive Examination-Revised total score;

${ }^{b}$ The California List A Learning trials 1-5 total score;

${ }^{c}$ The California Verbal Learning Test (CVLT) Short Delay Free Recall;

'The CVLT Short Delay Cued Recall;

e The CVLT Long Delay Free Recall;

${ }^{f}$ The CVLT Long Delay Cued Recall;

${ }^{g}$ CVLT discriminability;

${ }^{\text {h}}$ Follow up;

${ }^{i}$ Apolipoprotein E \&4.

women on most of the DXA measures. The differences are presented in Table 2 with respect to gender and APOE $\varepsilon 4$ status. $A P O E \& 4$ status and GDS (depression level) were not significantly associated with any of the DXA or cognitive variables.

\section{CORRELATIONS}

Table 3 presents the correlations between DXA, adiposity, and cognitive measures at baseline and follow up. Premorbid IQ (as measured using the CCRT) was not associated with any of 
Table 3 | Associations between Dual-energy X-ray absorptiometry (DXA), baseline, and follow up cognitive measures (2-tailed).

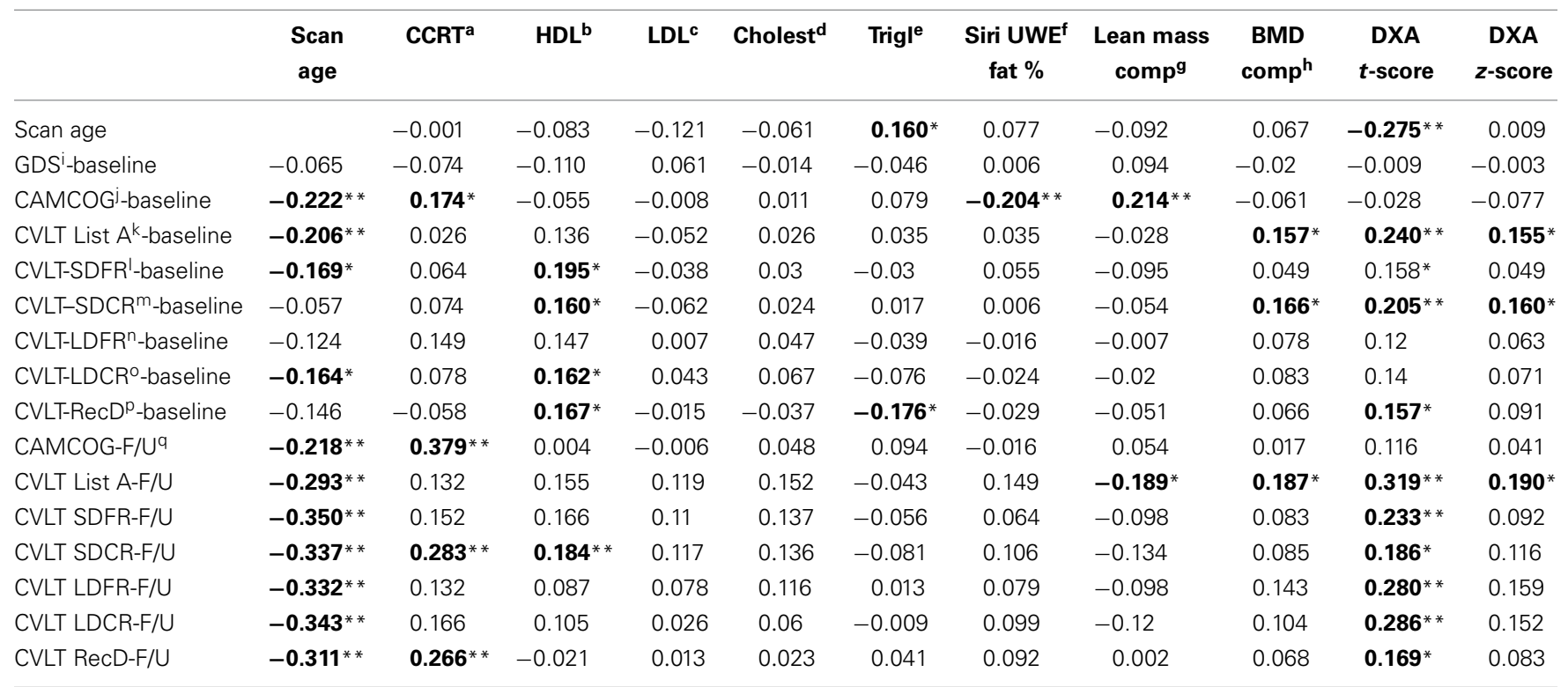

${ }^{*} p<0.05 ;{ }^{*} p<0.011$

${ }^{a}$ Cambridge Contextual Reading Test;

${ }^{b}$ High-density lipoprotein;

cLow-density lipoprotein:

${ }^{d}$ Cholesterol;

eTriglyceride;

${ }^{f}$ Underwater weight equivalents,

${ }^{g}$ Lean mass composite score included the DXA Lean mass Z scores for Midriff, Pelvis, Left leg, Right leg, Left arm, and Right arm divided by six;

${ }^{h}$ Bone Mineral Density Composite Score included the Z scores for DXA Spine L2-L4, Femoral Neck, and trochanter divided by three;

iGeriatric Depression Scale;

jThe Cambridge Cognitive Examination-Revised total score;

${ }^{k}$ The California Verbal Learning Test (CVLT) List A Learning trials 1-5 total score;

'The CVLT Short Delay Free Recall;

m The CVLT Short Delay Cued Recall;

${ }^{n}$ The CVLT Long Delay Free Recall;

oThe CVLT Long Delay Cued Recall;

${ }^{\circ}$ The CVLT discriminability;

${ }^{a}$ Follow up.

the DXA measures, but was a significant correlate of CAMCOG Baseline and FU scores, and CVLT SDCR and RecD follow up scores. GDS score was also not significantly associated with any of the DXA or other biomarkers and therefore was not included as a covariate in regression analyses. However, we found significant associations between overall cognitive status, as measured by CAMCOG- $\mathrm{R}$, and various DXA measures. A significant, negative association was found between baseline CAMCOG-R and Siri UWE $(r=-0.204, p<0.01)$. Lean mass and BMD composite scores were positively associated with verbal learning (CVLT scores) and general cognitive functioning (CAMCOG-R) (Table 3) and therefore included in the subsequent regression analyses. HDL was significantly associated with baseline CVLT subscales including, SDFR $(r=$ $0.195, p<0.05)$, SDCR $(r=0.160, p<0.05)$, and LDCR $(r=$ $0.162, p<0.05)$ but not with the CVLT follow up results except for SDCR $(r=0.184, p<0.05)$. Interestingly, HDL was negatively associated with the total perseveration score for List A learning trials $1-5(r=-0.160, p<0.05)$, indicating that greater perseveration was associated with lower HDL.

\section{CLINICAL AND COGNITIVE DATA}

Clinical and cognitive data were available for all participants. The mean clinical and cognitive scores for all participants are outlined in Tables 1, 2. The means for all neuropsychological measures were within expected age-related norms (Yesavage et al., 1982; Delis et al., 2000). We did not find any significant differences between APOE $\varepsilon 4$ carriers and non-carriers on any of the DXA or neuropsychological measures. Depression was not significantly associated with any of the DXA measures (Table 3) or with cognitive measures at baseline or follow up.

\section{REGRESSION ANALYSIS}

A series of hierarchical multiple regressions was conducted to examine the associations between DXA and adiposity measures 
and cognitive test results whilst controlling for the effects of relevant covariates at both baseline and follow up assessments. As discussed, demographic factors including age, gender, APOE $\varepsilon 4$ carriage, and premorbid IQ (as measured using the CCRT) were entered in Step 1. In Step 2, variables derived from DXA were entered into the model including the Siri UWE Fat percentage, Lean mass composite score, and the BMD composite score.

\section{COGNITIVE RESULTS}

The DXA scores in our two-step hierarchical regression model were not significantly associated with general cognitive functioning at baseline $\left(R^{2}\right.$-change $=0.007 ; F$ change $=0.359$, $p=0.782)$ or follow up $\left(R^{2}\right.$-change $=0.002 ; F$ change $=$ $0.110, p=0.954)$. In predicting baseline CVLT List A trials $1-5$, the first model containing covariates only was not significant $\left[F_{(4,141)}=2.014, p=0.096, R^{2}=0.054\right]$. However, the second model was significant $\left[F_{(7,138)}=2.359, p<0.05\right.$, $\left.R^{2}=0.107\right]$. Specifically, as seen in Table 4, the addition of the DXA variables provided unique predictive variance $\left(R^{2}\right.$ change $=0.53$ ). Higher $\mathrm{BMD}$ was significantly associated with higher learning scores, and there was a trend for lower Lean mass composite score $(B=-0.644 ; t=-1.926 ; p=$ 0.056).

Baseline CVLT discriminability (recognition) performance was not significantly predicted by the first model containing covariates only $\left[F_{(4,140)}=1.115, p=0.352, R^{2}=0.031\right.$, but the second model was significant, $F_{(7,137)}=2.422, p<0.05, R^{2}=$

Table 4 | Hierarchical linear regression predicting baseline CVLT List A trials 1-5 total score from lean mass and bone density composite scores.

\begin{tabular}{|c|c|c|c|c|c|}
\hline & $B$ & $\begin{array}{l}\text { Std. } \\
\text { error }\end{array}$ & $\beta$ & Adj. $R^{2}$ & $\Delta R^{2}$ \\
\hline Step 1 & & & & 0.027 & 0.054 \\
\hline Age & -0.247 & 0.097 & $-0.209 *$ & & \\
\hline Gender & 0.558 & 1.931 & 0.024 & & \\
\hline APOE $\varepsilon 4$ status & -2.413 & 1.849 & -0.107 & & \\
\hline $\mathrm{CCRT}^{\mathrm{a}}$ & 0.062 & 0.185 & 0.028 & & \\
\hline Step 2 & & & & 0.062 & $0.053^{*}$ \\
\hline Age & -0.322 & 0.101 & $-0.272 * *$ & & \\
\hline Gender & -5.201 & 3.629 & -0.221 & & \\
\hline APOE $\varepsilon 4$ Status & -2.457 & 1.837 & -0.109 & & \\
\hline CCRT & 0.073 & 0.183 & 0.032 & & \\
\hline Siri UWE ${ }^{b}$ fat $\%$ & 0.074 & 0.132 & 0.059 & & \\
\hline Lean mass comp score ${ }^{c}$ & -0.644 & 0.334 & $-0.274^{\#}$ & & \\
\hline BMD comp score ${ }^{d}$ & 3.123 & 1.138 & $0.252^{* *}$ & & \\
\hline
\end{tabular}

${ }^{*} p<0.05 ;{ }^{*} p<0.01 ; \# p<0.10$ (trend);

${ }^{a}$ Cambridge Contextual Reading Test;

bunderwater weight equivalents;

cLean mass composite score included the DXA Lean mass Z scores for Midriff, Pelvis, Left leg, Right leg, Left arm, and Right arm divided by six;

${ }^{d}$ Bone Mineral Density Composite Score included the $Z$ scores for DXA Spine L2-L4, Femoral Neck, and trochanter divided by three.
0.110]. Both lean mass composite score and BMD were significant predictors of RecDisc in the final model, with higher BMD and lower lean mass being associated with better performance (Table 5). Adding DXA variables contributed an additional 8\% of predictive variance.

CVLT List A learning at follow-up was also significantly predicted by DXA variables. The first model containing covariates only was significant $\left[F_{(4,124)}=6.005, p<0.001\right]$. The second model was also significant $\left[F_{(7,121)}=5.375, p<0.001, R^{2}=\right.$ $0.237]$. Specifically, the Lean mass composite score $(t=-2.297$, $p<0.05)$, and BMD composite score $(t=3.351, p<0.001)$ were significant predictors (Table 6), accounting for an additional $8 \%$ of variance.

Due to significant differences between men and women on DXA measures, we examined the interaction of gender and DXA measures (i.e., Siri UWE Fat \%, lean mass composite score, and BMD composite score) in predicting cognitive functions. The cognitive functions examined here included baseline and follow up CAMCOG-R, List A learning trials 1-5, SDFR, SDCR, LDCR, LDFR, and Recognition Disc results. Interestingly, we did not find any significant results for these interactions except for Gender $X \mathrm{BMD}$ on the follow up LDFR $\left[R^{2}\right.$-change = $0.052 ; F$ change $=2.707, p=0.048 ; F_{(10,118)}=3.944, p<$ $0.001 ; \mathrm{B}=-1.302, \mathrm{SE}=0.606$, Beta $=-0.698 ; t=-2.150, p<$ $0.034]$. Further analysis indicated that higher BMD was significantly associated with better follow up LDFR in men, but not in women $(r=0.459, p<0.002$ and $r=-0.039, p=0.707$, respectively).

Table 5 | Hierarchical linear regression predicting baseline CVLT discriminability score from lean mass and bone density composite scores.

$\begin{array}{lllll}\text { Std. } & \beta & \text { Adj. } R^{2} & \Delta R^{2} \\ \text { error } & & \end{array}$

$\begin{array}{llllll}\text { Step 1 } & & & & 0.003 & 0.031 \\ \text { Age } & -0.155 & 0.086 & -0.150 & & \\ \text { Gender } & -1.685 & 1.715 & -0.082 & & \\ \text { APOE } \varepsilon 4 \text { status } & -0.238 & 1.641 & -0.012 & & \\ \text { CCRT }^{\mathrm{a}} & -0.113 & 0.165 & -0.057 & & \end{array}$

Step 2

Age

Gender

$\begin{array}{rrr}-0.251 & 0.088 & -0.243^{*} *\end{array}$

APOE $\varepsilon 4$ status $\quad-0.460 \quad 1.609-0.023$

$\begin{array}{llll}\text { CCRT } & -0.089 & 0.160 & -0.045\end{array}$

Siri UWE ${ }^{b}$ fat \% $\quad 0.104 \quad 0.115 \quad 0.095$

Lean mass comp score ${ }^{c} \quad-0.970 \quad 0.293 \quad-0.471^{* * *}$

$\begin{array}{llll}\text { BMD comp score }^{\mathrm{d}} & 2.409 & 0.996 & 0.222^{*}\end{array}$ 
While the current study furthers our understanding of the relationship between age-related memory capacities and BMD, the cohort size and the very wide age range represent the main limitations. In addition, it would be advantageous to have baseline and follow up DXA results, and general morbidity and physical activity to examine the potential differences with negative changes in the DXA results in terms of memory and other cognitive functions. Additionally, examining the sex hormones will add value to a longitudinal study investigating the relationship between DXA scores and cognitive functions.

Further research is warranted investigating the relationship between BMD and cognitive dysfunctions in various dementia patient groups and in preclinical stages, and the potential mechanisms underlying these relationships. Such research could significantly improve our knowledge of the association between bone mineral content and higher cortical capabilities, and the relevance of these factors in aging and dementia. Future research may investigate the relationship between BMD and cognitive decline in aging while controlling for the effects of general mobility and current/previous physical activity as contributing factors.

The non-significant relationship between APOE alleles carriage, $\mathrm{BMD}$ and cognition in this study may indicate differential contributing pathways for genetic vs. lifestyle factors. Of course, we requires further research in a larger cohort where various genes identified as contributing to dementia risk can be examined in relation to $\mathrm{BMD}$ and cognitive decline measures.

\section{AUTHOR CONTRIBUTIONS}

Study design: HRS, KAB, JKF, SML, and RNM. Study conduct: HRS, KAB, MAR, MH, KT, GM, AP, JKF. Data collection: HRS, KAB, MAR, AP, GM. Data analysis: HRS, KAB, SSD, MW. Data interpretation: HRS, KAB, TS, RSB. Drafting manuscript: HRS, KAB. Revising manuscript content: MW, RSB, SRRS, JB, AC, MW, BMB, SMB, KT, TS, PDM, JKF, IJM, NLL, FM, SML. Approving final version of manuscript: HRS, KAB, MW, SSD, FM, NLL, and RNM. HRS, SSD, MW, and RNM take responsibility for the integrity of the data analysis.

\section{ACKNOWLEDGMENTS}

The WA Memory Study was supported by a grant from the National Health and Medical Research Council (NHMRC) of Australia (Grant Number: 324100) to RNM.

\section{REFERENCES}

Australian Institute of Health and Welfare. (2007). Dementia in Australia: National Data Analysis and Development. Canberra, ACT: Australian Institute of Health and Welfare, 315.

Bagger, Y. Z., Tanko, L. B., Alexandersen, P., Qin, G., and Christiansen, C. (2004). The implications of body fat mass and fat distribution for cognitive function in elderly women. Obes. Res. 12, 1519-1526. doi: 10.1038/oby.2004.189

Banks, L. M., Lees, B., MacSweeney, J. E., and Stevenson, J. C. (1994). Effect of degenerative spinal and aortic calcification on bone density measurements in post-menopausal women: links between osteoporosis and cardiovascular disease? Eur. J. Clin. Invest. 24, 813-817. doi: 10.1111/j.1365-2362.1994.tb02024.x

Bates, K. A., Sohrabi, H. R., Rodrigues, M., Beilby, J., Dhaliwal, S. S., Taddei, K., et al. (2009). Association of cardiovascular factors and Alzheimer's disease plasma amyloid-beta protein in subjective memory complainers. J. Alzheimers Dis. 17, 305-318. doi: 10.3233/JAD-2009-1050

Beardsall, L. (1998). Development of the Cambridge Contextual Reading Test for improving the estimation of premorbid verbal intelligence in older persons with dementia. Br. J. Clin. Psychol. 37(Pt 2), 229-240. doi: 10.1111/j.20448260.1998.tb01297.x

Beck, I. R., Gagneux-Zurbriggen, A., Berres, M., Taylor, K. I., and Monsch, A. U. (2012). Comparison of verbal episodic memory measures: consortium to establish a registry for Alzheimer's disease-Neuropsychological Assessment Battery (CERAD-NAB) versus California Verbal Learning Test (CVLT). Arch. Clin. Neuropsychol. 27, 510-519. doi: 10.1093/arclin/acs056

Brooks, B. L., Weaver, L. E., and Scialfa, C. T. (2006). Does impaired executive functioning differentially impact verbal memory measures in older adults with suspected dementia? Clin. Neuropsychol. 20, 230-242. doi: 10.1080/13854040590947461

Brownbill, R. A., and Ilich, J. Z. (2004). Cognitive function in relation with bone mass and nutrition: cross-sectional association in postmenopausal women. BMC Womens Health 4:2. doi: 10.1186/1472-6874-4-2

Brown, S. A., and Sharpless, J. L. (2004). Osteoporosis: an under-appreciated complication of diabetes. Clin. Diabetes 22, 10-20. doi: 10.2337/diaclin.22.1.10

Butterfield, D. A., Griffin, S., Munch, G., and Pasinetti, G. M. (2002). Amyloid betapeptide and amyloid pathology are central to the oxidative stress and inflammatory cascades under which Alzheimer's disease brain exists. J. Alzheimers Dis. 4, 193-201.

Clarnette, R. M., Almeida, O. P., Forstl, H., Paton, A., and Martins, R. N. (2001). Clinical characteristics of individuals with subjective memory loss in Western Australia: results from a cross-sectional survey. Int. J. Geriatr. Psychiatry 16, 168-174. doi: 10.1002/1099-1166(200102)16:2<168::AID-GPS291>3.0. CO;2-D

Corder, E. H., Saunders, A. M., Strittmatter, W. J., Schmechel, D. E., Gaskell, P. C., Small, G. W., et al. (1993). Gene dose of apolipoprotein E type 4 allele and the risk of Alzheimer's disease in late onset families. Science 261, 921-923. doi: 10.1126/science.8346443

Crockett, J. C., Rogers, M. J., Coxon, F. P., Hocking, L. J., and Helfrich, M. H. (2011). Bone remodelling at a glance. J. Cell Sci. 124, 991-998. doi: 10.1242/jcs. 063032

Delis, D. C., Freeland, J., Kramer, J. H., and Kaplan, E. (1988). Integrating clinical assessment with cognitive neuroscience: construct validation of the California Verbal Learning Test. J. Consult. Clin. Psychol. 56, 123-130. doi: 10.1037/0022006X.56.1.123

Delis, D. C., Kramer, J. H., Kaplan, A., and Ober, N. A. (2000). CVLT-II California Verbal Learning Test Manual Adult Version, 2nd Edn. Oxford: Harcourt Assessment Inc.

den Uyl, D., Nurmohamed, M. T., van Tuyl, L. H. D., Raterman, H. G., and Lems, W. F. (2011). (Sub) clinical cardiovascular disease is associated with increased bone loss and fracture risk; a systematic review of the association between cardiovascular disease and osteoporosis. Arthritis Res. Ther. 13:R5. doi: 10.1186/ar3224

Di Carlo, M., Giacomazza, D., and San Biagio, P. L. (2012). Alzheimer's disease: biological aspects, therapeutic perspectives and diagnostic tools. J. Phys. Condens. Matter 24:244102. doi: 10.1088/0953-8984/24/24/244102

Dimic, A., Popovic, M. R., Tasic, I., Djordjevic, D., Stojanovic, S., Stamenkovic, B., et al. (2012). Relation between bone density and certain parameters of lipid status in postmenopausal women. Cent. Eur. J. Med. 7, 642-649. doi: 10.2478/s11536-012-0044-6

Duff, K., Schoenberg, M. R., Scott, J. G., and Adams, R. L. (2005). The relationship between executive functioning and verbal and visual learning and memory. Arch. Clin. Neuropsychol. 20, 111-122. doi: 10.1016/j.acn.2004.03.003

Eimar, H., Tamimi, I., Murshed, M., and Tamimi, F. (2013). Cholinergic regulation of bone. J. Musculoskelet. Neuronal Interact. 13, 124-132.

Farhat, G. N., and Cauley, J. A. (2008). The link between osteoporosis and cardiovascular disease. Clin. Cases Miner. Bone Metab. 5, 19-34.

Farhat, G. N., Newman, A. B., Sutton-Tyrrell, K., Matthews, K. A., Boudreau, R., Schwartz, A. V., et al. (2007). The association of bone mineral density measures with incident cardiovascular disease in older adults. Osteoporos. Int. 18, 999-1008. doi: 10.1007/s00198-007-0338-8

Finkelstein, J. S., Brockwell, S. E., Mehta, V., Greendale, G. A., Sowers, M. R., Ettinger, B., et al. (2008). Bone mineral density changes during the menopause transition in a multiethnic cohort of women. J. Clin. Endocrinol. Metab. 93, 861-868. doi: 10.1210/jc.2007-1876

Folstein, M. F., Folstein, S. E., and McHugh, P. R. (1975). “Mini-mental state.” A practical method for grading the cognitive state of patients for the clinician. $J$. Psychiatr. Res. 12, 189-198. doi: 10.1016/0022-3956(75)90026-6 
Fox, M., Berzuini, C., and Knapp, L. A. (2013). Cumulative estrogen exposure, number of menstrual cycles, and Alzheimer's risk in a cohort of British women. Psychoneuroendocrinology 38, 2973-2982. doi: 10.1016/j.psyneuen.2013.08.005

Glenner, G. G., and Wong, C. W. (1984). Alzheimer's disease: initial report of the purification and characterization of a novel cerebrovascular amyloid protein. Biochem. Biophys. Res. Commun. 120, 885-890. doi: 10.1016/S0006291X(84)80190-4

Golby, A., Silverberg, G., Race, E., Gabrieli, S., O’Shea, J., Knierim, K., et al. (2005). Memory encoding in Alzheimer's disease: an fMRI study of explicit and implicit memory. Brain 128(Pt 4), 773-787. doi: 10.1093/brain/awh400

Guerra, R. S., Amaral, T. F., Marques, E., Mota, J., and Restivo, M. T. (2010). Accuracy of Siri and Brozek equations in the percent body fat estimation in older adults. J. Nutr. Health Aging 14, 744-748. doi: 10.1007/s12603-010-0112-z

Gustafson, D. R. (2012). Adiposity and cognitive decline: underlying mechanisms. J. Alzheimers Dis. 30, S97-S112. doi: 10.3233/JAD-2012-120487

Gustafson, D. R., and Luchsinger, J. A. (2013). High adiposity: risk factor for dementia and Alzheimer's disease? Alzheimers Res. Ther. 5:57. doi: 10.1186/alzrt221

Haberland, M., Schilling, A. F., Rueger, J. M., and Amling, M. (2001). Brain and bone: central regulation of bone mass. A new paradigm in skeletal biology. J. Bone Joint Surg. Am. 83, 1871-1876.

Hill, B. D., Alosco, M., Bauer, L., and Tremont, G. (2012). The relation of executive functioning to CVLT-II learning, memory, and process indexes. Applied neuropsychology Adult. 19, 198-206. doi: 10.1080/09084282.2011.643960

Hixson, J. E., and Vernier, D. T. (1990). Restriction isotyping of human apolipoprotein E by gene amplification and cleavage with HhaI. J. Lipid Res. 31, 545-548.

Holland, D., Desikan, R. S., Dale, A. M., and McEvoy, L. K. (2013). Higher rates of decline for women and apolipoprotein E\&4 carriers. AJNR Am. J. Neuroradiol. 34, 2287-2293. doi: 10.3174/ajnr.A3601

Isacson, O., Seo, H., Lin, L., Albeck, D., and Granholm, A. C. (2002). Alzheimer's disease and Down's syndrome: roles of APP, trophic factors and ACh. Trends Neurosci. 25, 79-84. doi: 10.1016/S0166-2236(02)02037-4

Kanaya, A. M., Lindquist, K., Harris, T. B., Launer, L., Rosano, C., Satterfield, S., et al. (2009). Total and regional adiposity and cognitive change in older adults: the Health, Aging and Body Composition (ABC) study. Arch. Neurol. 66, 329-335. doi: 10.1001/archneurol.2008.570

Kanis, J. A. (2002). Diagnosis of osteoporosis and assessment of fracture risk. Lancet 359, 1929-1936. doi: 10.1016/S0140-6736(02)08761-5

Karsenty, G., and Oury, F. (2010). The central regulation of bone mass, the first link between bone remodeling and energy metabolism. J. Clin. Endocrincol. Metab. 95, 4795-4801. doi: 10.1210/jc.2010-1030

Kerwin, D. R., Gaussoin, S. A., Chlebowski, R. T., Kuller, L. H., Vitolins, M., Coker, L. H., et al. (2011). Interaction between body mass index and central adiposity and risk of incident cognitive impairment and dementia: results from the Women's Health Initiative Memory Study. J. Am. Geriatr. Soc. 59, 107-112. doi: 10.1111/j.1532-5415.2010.03219.x

Kohlmeier, M., Salomon, A., Saupe, J., and Shearer, M. J. (1996). Transport of vitamin K to bone in humans. J. Nutr. 126(4 Suppl.), 1192S-1196S.

Laws, S. M., Clarnette, R. M., Taddei, K., Martins, G., Paton, A., Hallmayer, J., et al. (2002). APOE 4 and APOE 491A polymorphisms in individuals with subjective memory loss. Mol. Psychiatry 7, 768-775. doi: 10.1038/sj.mp.4001083

Levine, M. E., and Crimmins, E. M. (2012). Sarcopenic obesity and cognitive functioning: the mediating roles of insulin resistance and inflammation? Curr. Gerontol. Geriatr. Res. 2012:826398. doi: 10.1155/2012/826398

Lieb, W., Beiser, A. S., Vasan, R. S., Tan, Z. S., Au, R., Harris, T. B., et al. (2009). Association of plasma leptin levels with incident Alzheimer disease and MRI measures of brain aging. JAMA 302, 2565-2572. doi: 10.1001/jama.2009.1836

Luchsinger, J. A., and Mayeux, R. (2007). Adiposity and Alzheimer's disease. Curr. Alzheimer Res. 4, 127-134. doi: 10.2174/156720507780362100

Luchsinger, J. A., Patel, B., Tang, M. X., Schupf, N., and Mayeux, R. (2007). Measures of adiposity and dementia risk in elderly persons. Arch. Neurol. 64, 392-398. doi: 10.1001/archneur.64.3.392

Lui, L. Y., Stone, K., Cauley, J. A., Hillier, T., and Yaffe, K. (2003). Bone loss predicts subsequent cognitive decline in older women: the study of osteoporotic fractures. J. Am. Geriatr. Soc. 51, 38-43. doi: 10.1034/j.1601-5215.2002.51007.x

Mahley, R. W., Nathan, B. P., and Pitas, R. E. (1996). Apolipoprotein, E. Structure, function, and possible roles in Alzheimer's disease. Ann. N.Y. Acad. Sci. 777, 139-145. doi: 10.1111/j.1749-6632.1996.tb34412.x
Masters, C. L., Multhaup, G., Simms, G., Pottgiesser, J., Martins, R. N., and Beyreuther, K. (1985). Neuronal origin of a cerebral amyloid: neurofibrillary tangles of Alzheimer's disease contain the same protein as the amyloid of plaque cores and blood vessels. EMBO J. 4, 2757-2763.

Maylor, E. A., Reimers, S., Choi, J., Collaer, M. L., Peters, M., and Silverman, I. (2007). Gender and sexual orientation differences in cognition across adulthood: age is kinder to women than to men regardless of sexual orientation. Arch. Sex. Behav. 36, 235-249. doi: 10.1007/s10508-006-9155-y

Norton, S., Matthews, F. E., Barnes, D. E., Yaffe, K., and Brayne, C. (2014). Potential for primary prevention of Alzheimer's disease: an analysis of population-based data. Lancet Neurol. 13, 788-794. doi: 10.1016/S1474-4422(14)70136-X

Parhami, F., Garfinkel, A., and Demer, L. L. (2000). Role of lipids in osteoporosis. Arterioscler. Thromb. Vasc. Biol. 20, 2346-2348. doi: 10.1161/01.ATV.20.11.2346

Parhami, F., Morrow, A. D., Balucan, J., Leitinger, N., Watson, A. D., Tintut, Y., et al. (1997). Lipid oxidation products have opposite effects on calcifying vascular cell and bone cell differentiation - A possible explanation for the paradox of arterial calcification in osteoporotic patients. Arterioscler. Thromb. Vasc. Biol. 17, 680-687. doi: 10.1161/01.ATV.17.4.680

Price, P. A., June, H. H., Buckley, J. R., and Williamson, M. K. (2001). Osteoprotegerin inhibits artery calcification induced by warfarin and by vitamin D. Arterioscler. Thromb. Vasc. Biol. 21, 1610-1616. doi: 10.1161/hq1001. 097102

Prince, M., Bryce, R., Albanese, E., Wimo, A., Ribeiro, W., and Ferri, C. P. (2013). The global prevalence of dementia: a systematic review and metaanalysis. Alzheimers Dement. 9, 63-75.e2. doi: 10.1016/j.jalz.2012.11.007

Qiu, C. X., Xu, W. L., Winblad, B., and Fratiglioni, L. (2010). Vascular risk profiles for dementia and Alzheimer's disease in very old people: a population-based longitudinal study. J. Alzheimers Dis. 20, 293-300. doi: 10.3233/JAD-2010-1361

Roses, A. D. (1997). Apolipoprotein, E., a gene with complex biological interactions in the aging brain. Neurobiol Dis. 4, 170-185. doi: 10.1006/nbdi.1997.0161

Roth, M., Huppert, F. A., Mountjoy, C. Q., and Tym, E. (1998). CAMDEX-R: The Cambridge Examination for Mental Disorders of the Elderly-Revised. Cambridge, UK: Cambridge University Press.

Rothman, M. S., Arciniegas, D. B., Filley, C. M., and Wierman, M. E. (2007). The neuroendocrine effects of traumatic brain injury. J. Neuropsychiatry Clin. Neurosci. 19, 363-372. doi: 10.1176/jnp.2007.19.4.363

Sarkis, K. S., Martini, L. A., Szejnfeld, V. L., and Pinheiro, M. M. (2012). Low fatness, reduced fat intake and adequate plasmatic concentrations of LDL-cholesterol are associated with high bone mineral density in women: a cross-sectional study with control group. Lipids Health Dis. 11:37. doi: 10.1186/1476-511X-11-37

Saunders, A. M., Strittmatter, W. J., Schmechel, D., George-Hyslop, P. H., PericakVance, M. A., Joo, S. H., et al. (1993). Association of apolipoprotein E allele $\varepsilon 4$ with late-onset familial and sporadic Alzheimer's disease. Neurology 43, 1467-1472. doi: 10.1212/WNL.43.8.1467

Schoofs, M. W. C. J., van der Klift, M., Hofman, A., van Duijn, C. M., Stricker, B. H. C., A. P., Pols, H., et al. (2004). ApoE gene polymorphisms, BMD, and fracture risk in elderly men and women: the Rotterdam Study. J. Bone Miner. Res. 19, 1490-1496. doi: 10.1359/JBMR.040605

Sohrabi, H. R., Bates, K. A., Rodrigues, M., Taddei, K., Martins, G., Laws, S. M., et al. (2009). The relationship between memory complaints, perceived quality of life and mental health in apolipoprotein E\&4 carriers and non-carriers. J. Alzheimers Dis. 17, 69-79. doi: 10.3233/JAD-2009-1018

Takeda, S. (2009). Osteoporosis: a neuroskeletal disease? Int. J. Biochem. Cell Biol. 1, 455-459. doi: 10.1016/j.biocel.2008.08.002

Tan, Z. S., Seshadri, S., Beiser, A., Zhang, Y., Felson, D., Hannan, M. T., et al. (2005). Bone mineral density and the risk of Alzheimer disease. Arch. Neurol. 62, 107-111. doi: 10.1001/archneur.62.1.107

Twamley, E. W., Ropacki, S. A., and Bondi, M. W. (2006). Neuropsychological and neuroimaging changes in preclinical Alzheimer's disease. J. Int. Neuropsychol. Soc. 12, 707-735. doi: 10.1017/S1355617706060863

Van Loan, M. D., and Mayclin, P. L. (1992). Body composition assessment: dualenergy X-ray absorptiometry (DEXA) compared to reference methods. Eur. J. Clin. Nutr. 46, 125-130.

Verdile, G., Fuller, S., Atwood, C. S., Laws, S. M., Gandy, S. E., and Martins, R. N. (2004). The role of beta amyloid in Alzheimer's disease: still a cause of everything or the only one who got caught? Pharmacol. Res. 50, 397-409. doi: 10.1016/j.phrs.2003.12.028 
Vickland, V., Morris, T., Draper, B., Low, L., and Brodaty, H. (2012). Modelling the Impact of Interventions to Delay the Onset of Dementia in Australia. A report for Alzheimer's Australia. Alzheimer's Australia.

Weisgraber, K. H. (1994). Apolipoprotein E: structure-function relationships. Adv. Protein Chem. 45, 249-302. doi: 10.1016/S0065-3233(08)60642-7

White, K. G., and Ruske, A. C. (2002). Memory deficits in Alzheimer's disease: the encoding hypothesis and cholinergic function. Psychon. Bull. Rev. 9, 426-437. doi: 10.3758/BF03196301

Whitmer, R. A., Gustafson, D. R., Barrett-Connor, E., Haan, M. N., Gunderson, E. P., and Yaffe, K. (2008). Central obesity and increased risk of dementia more than three decades later. Neurology 71, 1057-1064. doi: 10.1212/01.wnl.0000306313.89165.ef

W.H.O. (1994). Assessment of Fracture Risk and its Application to Screening for Postmenopausal Osteoporosis. Report of a WHO Study Group. World Health Organization.

Wirths, O., Multhaup, G., and Bayer, T. A. (2004). A modified beta-amyloid hypothesis: intraneuronal accumulation of the beta-amyloid peptide-the first step of a fatal cascade. J. Neurochem. 91, 513-520. doi: 10.1111/j.14714159.2004.02737.x

Wolf, P. A., Beiser, A., Elias, M. F., Au, R., Vasan, R. S., and Seshadri, S. (2007). Relation of obesity to cognitive function: importance of central obesity and synergistic influence of concomitant hypertension. The Framingham Heart Study. Curr. Alzheimer Res. 4, 111-116. doi: 10.2174/156720507780362263

Yaffe, K., Browner, W., Cauley, J., Launer, L., and Harris, T. (1999). Association between bone mineral density and cognitive decline in older women. J. Am. Geriatr. Soc. 47, 1176-1182.

Yaffe, K., Sawaya, G., Lieberburg, I., and Grady, D. (1998). Estrogen therapy in postmenopausal women: effects on cognitive function and dementia. JAMA 279, 688-695. doi: 10.1001/jama.279.9.688

Yang, R., and Barouch, L. A. (2007). Leptin signaling and obesity: cardiovascular consequences. Circ. Res. 101, 545-559. doi: 10.1161/CIRCRESAHA.107.156596

Yesavage, J. A., Brink, T. L., Rose, T. L., Lum, O., Huang, V., Adey, M., et al. (1982). Development and validation of a geriatric depression screening scale: a preliminary report. J. Psychiatr. Res. 17, 37-49. doi: 10.1016/0022-3956(82) 90033-4

Yoon, D. H., Choi, S. H., Yu, J. H., Ha, J. H., Ryu, S. H., and Park, D. H. (2012). The relationship between visceral adiposity and cognitive performance in older adults. Age Ageing 41, 456-461. doi: 10.1093/ageing/afs018

Zhang, Y., Seshadri, S., Ellison, R. C., Heeren, T., and Felson, D. T. (2001). Bone mineral density and verbal memory impairment: Third National Health and Nutrition Examination Survey. Am. J. Epidemiol. 154, 795-802. doi: 10.1093/aje/154.9.795

Conflict of Interest Statement: RNM is the founder and owns stock in Alzhyme. HRS has received personal compensation for activities with Pfizer and Wyeth and currently with Takeda Pharmaceuticals. The authors declare that the research was conducted in the absence of any commercial or financial relationships that could be construed as a potential conflict of interest.

Received: 18 November 2014; accepted: 03 February 2015; published online: 18 February 2015.

Citation: Sohrabi HR, Bates KA, Weinborn M, Bucks RS, Rainey-Smith SR, Rodrigues MA, Bird SM, Brown BM, Beilby J, Howard M, Criddle A, Wraith M, Taddei K, Martins G, Paton A, Shah T, Dhaliwal SS, Mehta PD, Foster JK, Martins IJ, Lautenschlager NT, Mastaglia F, Laws SM and Martins RN (2015) Bone mineral density, adiposity, and cognitive functions. Front. Aging Neurosci. 7:16. doi: 10.3389/fnagi. 2015.00016

This article was submitted to the journal Frontiers in Aging Neuroscience.

Copyright (C) 2015 Sohrabi, Bates, Weinborn, Bucks, Rainey-Smith, Rodrigues, Bird, Brown, Beilby, Howard, Criddle, Wraith, Taddei, Martins, Paton, Shah, Dhaliwal, Mehta, Foster, Martins, Lautenschlager, Mastaglia, Laws and Martins. This is an open-access article distributed under the terms of the Creative Commons Attribution License (CC BY). The use, distribution or reproduction in other forums is permitted, provided the original author(s) or licensor are credited and that the original publication in this journal is cited, in accordance with accepted academic practice. No use, distribution or reproduction is permitted which does not comply with these terms. 